McClurg D, Bugge C, Elders A, Irshad T, Hagen S, Moore K, Buckley B \& Fader M (2019) Factors affecting continuation of clean intermittent catheterisation in people with multiple sclerosis: Results of the COSMOS mixed-methods study, Multiple Sclerosis Journal, Volume 25, issue 5, pp. 727-739. Copyright (C) Authors 2018. Reprinted by permission of SAGE Publications. 


\title{
Factors affecting continuation of clean intermittent catheterisation in people with multiple sclerosis: Results of the COSMOS mixed-methods study
}

Doreen McClurg, NMAHP RU, Glasgow Caledonian University, Glasgow, UK

Carol Bugge, Faculty of Health Sciences and Sport, University of Stirling, Stirling, UK

Andrew Elders, NMAHP RU, Glasgow Caledonian University, Glasgow, UK

Tasneem Irshad, NMAHP RU, Glasgow Caledonian University, Glasgow, UK

Suzanne Hagen, NMAHP RU, Glasgow Caledonian University, Glasgow, UK

Katherine N Moore, Faculty of Nursing, University of Alberta, Edmonton, AB, Canada

Brian Buckley, Division of Urology, Department of Surgery, Philippine General Hospital, Manila, Philippines

Mandy Fader, Health Sciences, University of Southampton, Southampton, UK/Continence and Skin Technology, University of Southampton, Southampton, UK

\begin{abstract}
Background: Clean Intermittent self-catheterisation $(\mathrm{CIC})$ is often recommended for people with MS.
\end{abstract}

Objectives: To determine the variables that affect continuation or discontinuation of the use of CIC.

Methods: A 3-part mixed method study (prospective longitudinal cohort $(n=56)$, longitudinal qualitative interviews $(n=20)$ and retrospective survey $(n=456)$ was undertaken which identified the variables that influenced clean intermittent selfcatheterisation continuation/discontinuation. The potential explanatory variables investigated in each study were the individual's age, gender, social

circumstances, number of urinary tract infections bladder symptoms, presence of comorbidity, stage of multiple sclerosis and years since diagnosis, as well as clean intermittent catheterization teaching method and intensity.

Results: For some people with multiple sclerosis the prospect of undertaking clean intermittent catheterisation is difficult and may take a period of time to accept before beginning the process of using clean intermittent catheterisation. Ongoing support from clinicians, support at home and a perceived improvement in symptoms such as nocturia were positive predictors of continuation. In many cases, the development of a urinary 
tract infection during the early stages of clean intermittent catheterisation use had a significant detrimental impact on continuation.

Conclusions: Procedures for reducing the incidence of urinary tract infection during the learning period (i.e. when being taught and becoming competent) should be considered, as well as the development of a tool to aid identification of a person's readiness to try clean intermittent catheterization.

\section{Background}

Multiple sclerosis is the most common neurological disease of young adults affecting more than 100,000 people in the UK in a ratio of $1: 3$ male to female. Lower urinary tract dysfunction is present in $75-80 \%$ of patients with MS and urinary incontinence in more than $50 \%$, and this is influenced by the duration and extent of disease. Patients most commonly report symptoms of urinary urgency, incontinence and frequency, as well as the inability to initiate voiding voluntarily. ${ }^{1}$

It is thought that clean intermittent catheterisation $(\mathrm{CIC})$ is advocated at some point in at least $25 \%$ of people with $\mathrm{MS}^{2}$ to assist in overall bladder management especially for those with voiding difficulties. In 2008 an expert panel produced a UK consensus document on the management of the bladder in people with MS (PwMS). They considered clean intermittent catheterisation $(\mathrm{ClC})$ to be of the greatest importance in the management of

neurogenic bladder dysfunction caused by $\mathrm{MS}^{3} \mathrm{CIC}$ is more effective than indwelling catheterisation for those who can perform it themselves or have a carer who can do so for them. ${ }^{4}$ It reduces the risk of common indwelling catheter-associated complications such as blockage, and reduces the need for equipment such as drainage bags. $\mathrm{CIC}$ may also reduce lower urinary tract symptoms (frequency, urgency and incontinence) between catheterisation, which can lead to improved self-care and independence, with fewer barriers to intimacy and sexual activities. ${ }^{5}$ However, $\mathrm{CIC}$ is not always taken up, or if tried is often discontinued in people with MS. ${ }^{6,7}$ The primary aim of this study was to identify the variables that affect continuation of use of clean intermittent catheterisation. 


\section{Methods}

Data were collected using mixed methods in order to improve the understanding of the variables involved in the decision to continue $\mathrm{CIC}^{8,9}$ and comprised three study components (Figure 1). We identified the most probable variables thought to influence $\mathrm{CIC}$ use through personal communication with experts in the field, including two of the authors of a subsequent paper, ${ }^{10}$ and consultation with a group of MS patients reaching consensus on the top 10 (Insert Figure 1). These 10 potential predictive variables were then targeted in our data collection - age, gender, social circumstances, number of urinary tract infections (UTIs), bladder symptoms, presence of co-morbid and or MS symptoms, stage of MS, years since diagnosis, teaching venue, teaching intensity.

\subsection{Prospective Longitudinal Cohort:}

Across 38 UK wide sites we recruited people with MS who had been newly referred to the continence service due to bladder symptoms over a 12 month period (May 2013 to April 2014). Baseline demographic and symptom characteristics are presented in Table 1 . The target sample size $(n=250)$ for the prospective longitudinal cohort study was based on a planned logistic regression analysis using the 10 independent variables mentioned above and shown in Table 2, and assuming that the proportion discontinuing with $\mathrm{CIC}$ was $40 \%$ following the sample size guidelines of Peduzzi et al $1996 .^{11}$

All participants completed disease-specific (ICIQ-LUTS, FLUTS) and general health status $(E Q-5 D)^{12}$ questionnaires at 4, 8 and 12 months after referral (Table 3). The ICIQ-FLUTS has 13 questions around severity of bladder symptoms with responses scoring from 0 least and 4 worse scenario ${ }^{13}$; and ICIQ-LUTSqol with 22 questions on impact on daily activities with 1 indicating least and 4 greatest impact. ${ }^{14}$ These questionnaires have Grade A validity, reliability and responsiveness which have been established in several nonneurogenic data sets. ${ }^{15,16}$ These were used as, at the time of study inception, they seemed the most appropriate questionnaires to assess severity of bladder symptoms and impact on daily activities. We had used these questionnaires in other populations and they have been also been used in the MS population. ${ }^{17,18}$ The EQ- $5 D^{12}$ is one of the most commonly used generic questionnaires to measure health-related quality of life self-assessment in 5 
dimensions as well as the EQ-VAS which records the subject's perceptions of their own current overall health and can be used to monitor changes with time.

The number of UTIs were as reported by the participant at telephone follow-up call and included all symptomatic UTIs for which antibiotics had been taken, but not necessarily laboratory-confirmed UTI (Table 4).

\section{Analysis}

Quantitative data analysis included summary statistics (proportions for categorical variables, means and SDs for continuous variables) describing, for all participants, the demographic characteristics and quality of life measures over the study time-points using responses provided by participants.

Participants were grouped into four categories based on their $\mathrm{CIC}$ use: $\mathrm{CIC}$ not recommended; $\mathrm{CIC}$ recommended but not started - resistors; CIC started but stopped - discontinuers; and $\mathrm{CIC}$ started and continuing at the one year follow-up - continuers. This paper focusses on the latter two groups only. Discontinuers and continuers were compared using Pearson chisquare tests for categorical variables and two-sample t-tests for continuous variables. Independent samples Mann-Whitney U-tests for were used for continuous data if the overall sample was not normally distributed (Shapiro-Wilk statistics were used to test normality). For outcomes collected at multiple time points (ie ICIQ FLUTS and LUTS), discontinuers and continuers were compared using repeated measures mixed models with restricted maximum likelihood, adjusting for age, sex, duration of symptoms, type of MS and general health status, with participant fitted as a random effect. Data collected at baseline and 12 months (i.e. EQ5D) were compared at 12 months using analysis of covariance adjusting for the same variables used in the repeated measures models along with the baseline measure. Binary logistic regression was used to identify if any of the fixed effects used in the repeated measures model are potential predictors of $\mathrm{CIC}$ continuation at 12 months. Linearity assumptions and normality of error distributions were examined by inspection of residual plots for the linear models, and the goodness of fit for the logistic model was examined with a HosmerLemeshow test. The significance level used was 5\%, and exact significance (rather than asymptotic significance) was reported to correct for small numbers of responses where applicable. Statistical analyses were performed using SPSS v21 (IBM, Armonk, NY). 


\subsection{Qualitative Interviews}

In-depth one-to-one interviews were undertaken with a purposive sample (variance based on age, gender, type of MS and disability level) of patients from the longitudinal cohort who had commenced $\mathrm{CIC}$ following referral to the continence service. We aimed for a sample of 20 people, which we anticipated would provide saturation of the concepts. ${ }^{19}$ Telephone interviews were conducted with each individual at two specific time-points ( 4 and 12 months after recruitment) by DM (a specialist physiotherapist and academic researcher). Interviews lasted between 30 and 45 minutes and were audio recorded. The focus of the interviews was to allow participants to discuss the variables they felt were important to continuation and discontinuation of $\mathrm{CIC}$.

All interviews were transcribed verbatim and data were analysed using Framework Analysis. $^{20}$ A subset of data were selected and three of the team (DM, AT, CB) familiarised themselves with the data. From that, an initial thematic framework was developed and applied across the data. The thematic framework was revisited and expanded as necessary when applied to the broader data set. Charts were developed around the key themes and compared to generate an understanding of the variables that affect continuation and discontinuation.

\subsection{On-line Survey}

An on-line survey was disseminated through the UK-based MS Registry portal (https://www.ukmsregister.org/). All members of the Registry (which is voluntary) are alerted by email to the availability of such research, and can access the portal to respond. An initial explanatory paragraph introduced the aims and methods of the study. The content and phrasing of the questions of this survey were informed by the qualitative interviews and the 10 potential predictor variables outlined earlier. (Appendix 3 ). Once accessed the survey gave a brief background to the study, ethical approval details and contact details of the researcher who could answer questions. Comparison of those who had continued using $\mathrm{CIC}$ and those who had discontinued was undertaken as per the analysis of the longitudinal study data. 


\section{Data Triangulation}

To develop the analysis and drive the action and impact of our research we have combined the methods using a triangulation protocol. ${ }^{21}$ Triangulation is a powerful technique that facilitates validation of data through cross verification from two or more sources. In particular, it refers to the application and combination of several research methods in the study of the same phenomenon. In this study we used the 10 regression variables and if they were identified in the longitudinal, qualitative and survey studies. If all three studies identified a particular variable as having an impact on ISC then this was Total Agreement, if only 2 this was Partial agreement.

\subsection{Ethical approval}

Ethical approval was granted by the West of Scotland REC 2 committee 11/AL/0117 on $24^{\text {th }}$ March 2011.

\section{RESULTS}

\section{Prospective Longitudinal Cohort}

From the 260 patients who were interested in taking part in the trial 224 participants completed the Consent form and 204 returned the base-line from 38 sites across the UK (Figure 2). This number fell short of the target sample size of 250. From the 204, 56 (27\%) commenced CIC (Continued and discontinued Groups) of these 13 (23\%) (Discontinued Group) stopped using $\mathrm{CIC}$ within the one-year follow-up, 6 within the first 4 months and the remaining 7 by month 8 . A further $13(6 \%)$ were recommended to commence $\mathrm{CIC}$ but chose not to do so. Ninety per cent of participants who did not commence CIC ( $n=119 / 148$ ) completed the Outcome questionnaires at 12 months, $100 \%$ of those that had commenced CIC (56/56) completed the questionnaires at 12 months. (Insert Figure 2)

Baseline demographic and symptom data are shown in Table 1. Comparative analysis has been used to compare (in this paper) those that commenced $\mathrm{CIC}$ and continued to those that commenced and discontinued.

There was no significant difference in the age, gender, severity of bladder symptoms or length of time since diagnosis at baseline between those that subsequently discontinued and those that continued CIC. As shown in Table 1 there was however a 
significant difference in the severity of symptoms related to MS $(\chi 2=10.923, d f=4$, $\mathrm{p}=0.0459$ ) with a greater proportion of discontinuers reporting problems of balance and fatigue (Table 1$)$.

Although not significantly different the majority of those that continued had been taught in clinic and had been seen 4-6 times compared to once or twice. Those that discontinued $\mathrm{CIC}$ were more likely to either live alone or be single parents compared to those that continued (Table 2). There were no significant differences in the Total LUTS and FLUTS scores between those that discontinued and those that continued at any time-point (Table 3).

More participants who continued with CIC had reported UTIs at baseline (51\%) compared with those who discontinued (23\%) (Chi square test $\mathrm{p}=0.064)$. There were significantly more UTIs at 8 months in those that discontinued $\mathrm{CIC}$ compared to those that continued (Chi Square test, $p=0.019$ ). (Table 4)

The logistic regression model showed no evidence of any independent risk variables significantly associated with discontinuation. (see model output in Appendix 2)

\section{Qualitative Longitudinal Interviews (See Appendix 1 for characteristics of participants)}

Twenty participants took part in the interviews all of whom started CIC; all 20 were interviewed at four months and 19 again at 12 months (39 interviews). There were 16 female and four male participants with a mean age of 53 years. Nine participants reported having Relapsing Remitting, four Primary Progressive and seven Secondary Progressive MS. Four participants did not use any aids when walking, seven used two sticks or rollator and nine primarily used wheelchairs/scooters. All interview participants self-catheterised (i.e. no carer involvement). At the one- year follow-up six participants had discontinued CIC. Brief findings from the analysis is reported below. Supportive qualitative information is available as supplementary data.

Demographic variables (age, gender and social circumstances)

There was no obvious difference in the demographic data between those that continued or discontinued

Teaching method, place, intensity and support 
Generally, starting $\mathrm{CIC}$ was suggested by the continence nurse with feelings often changing from being against trying to being ready to try.

\section{Bladder symptoms, including UTIs}

The two main benefits of $\mathrm{ClC}$ raised by participants were sleeping better at night, as a result of reduced nocturia, and being more confident and comfortable when going out/ going shopping.

\section{About MS (symptoms, type of MS, and time since diagnosis)}

Participants did not raise MS type or time since diagnosis when asked about variables that support continuation or discontinuation. There was a contrast between those who felt that by starting $\mathrm{CIC}$ it was giving in to the disease and losing control' whilst those that went on to continue using $\mathrm{CIC}$ stated that they 'felt more in control' being able to go out without worrying about accessing the toilets.

\section{On-line Survey (See Appendix 3 for Online Survey)}

Of the 11,000 registrants $2227(20 \%)$ responded to the survey; from the responders 454(20\%) had used CIC, of whom 167 (36\%) had then discontinued, and 291 (64\%) were continuing to use $\mathrm{CIC}$. The mean age of all responders was 47.5 (SD 11.4) years, with 1578 (71\%) females and $645(28 \%)$ male. (Table 5). There was no significant difference in the gender ratio or age of those who had stopped compared to those who continued to use $\mathrm{CIC}$ (Table 5). The average length of time that $\mathrm{CIC}$ was used by those that had stopped was 22 (SD 33) months, although 41/167 (25\%) had stopped within one month. The primary reasons for stopping CIC were: an increase in number of UTIs (51/167, 31\%); poor dexterity and or pain (35/167, 21\%); felt it was humiliating/degrading (20/167, $12 \%)$; no longer necessary due to improvement (16/167, 10\%); using CIC did not improve symptoms $(16 / 167,10 \%)$.

Negativity was the overwhelming feeling in both groups when CIC was first suggested with some expressing that it was the fear of 'loss of control' that was the worst thought. Improvements in symptoms of emptying, leakage, nocturia and frequency were the most often reported changes in symptoms in those that continued to use $\mathrm{CIC}$. In those 
that had discontinued more said their emptying, number of UTIs and QoL were worse. (Table 6).

\section{Data Triangulation (Figure 3)}

There was total agreement that age or gender or length of time since MS diagnosis did not impact on continuation of $\mathrm{CIC}$ but MS symptoms such as poor dexterity and frequency of UTIs did affect continuation. There was also total agreement that professional support and support at home were important for continuation. There was partial agreement that if there was significant improvements in symptoms (the qualitative and survey data) patients were more likely to continue with CIC. Two studies (partial agreement) agreed that the type of MS did not seem to impact on continuation; however the type of MS was not brought up in the interviews.

\section{Discussion}

Our research question i.e. 'what determines the continuity of ISC' was recognised to be complex from the outset and therefore a mixed methods was used with integration of findings using Data Triangulations 21,22. (Figure 3 Data Triangulation). Several important conclusions may be drawn from the information gathered about the ten variables we explored in detail in this study. Age, gender, type of MS and time since diagnosis had no effect on continuation with $\mathrm{CIC}$. Being taught at home was commonly preferred and there is a tentative suggestion that it may lead to a greater likelihood of continuing. Ongoing clinician support and family support at home both improved chances of continuing as did the reduction in nocturia experienced. UTI's during the initial period of use and poor balance, dexterity and fatigue were identified as leading to to greater discontinuation.

One key variable relates to the development, and perception of the development of UTIs. Most patients who stop $\mathrm{CIC}$ do so within the first 1-3 months and the occurrence of one or more UTIs during this period significantly impacts on continuation (See Figure 3).

This is an important finding as $\mathrm{CIC}$ has been shown to be efficacious in some populations e.g. spinal cord injuries where the main criteria for success is continence, ability to selfcatheterise and the preservation of kidney function. ${ }^{23} \mathrm{~A}$ reduction or an increase in 
the incidence of UTI can be difficult to determine due to poor definition of UTI, mixed study populations and different types of catheters. However, it would seem within the MS population any perceived increase is a predictive factor for discontinuation and there is evidence that during the learning period an infection may be more likely to develop. A more focussed strategy at this time may help with continuation and therefore the longerterm benefits may become more apparent to the patiet; for example, using a low-fidelity simulation may help to increase self-confidence ${ }^{25}$ and it may be worth exploring the potential benefits of using prophylactic antibiotics during the learning phase, however this needs to be further explored in relation to a possible increase resistance of urinary bacteria. ${ }^{26}$

Variables relating to the nature of MS as an illness (for example, poor balance and dexterity) and variables relating to the clinical and personal support available seemed to influence a person's readiness to undertake CIC. There appeared to be no correlation between the initial reaction to the suggestion of using $\mathrm{CIC}$ (accepting versus opposing) and continuation/discontinuation. However, the data also identified that the individual had to be "ready" - emotionally and physically - to be able to add another 'burden' to their daily life. For some there was a definite change of perception over time and for those in which benefit outweighed burden continuation was more usual but where this was not evident then discontinuation was more usual. It was evident that for many making the decision to start $\mathrm{CIC}$ was a major step and support for the individual with MS, both from the person teaching $\mathrm{CIC}$ and from their family members was important to encourage them to overcome both the practical issues and feelings of negativity. A recently published review on the quality of life of ISC users and their caregivers ${ }^{24}$ concluded that the QoL of patient's who use ISC can be determined by improvement of urinary symptoms and selfconfidence with emphasis on the importance of adequate professional support.

The current study has strengths and limitations. It is the first study that brings together prospective longitudinal quantitative and qualitative, and retrospective data relating to variables affecting continuation and discontinuation of $\mathrm{CIC}$ use in an $\mathrm{MS}$ population. However, recruitment was slower than expected and the reduced numbers of $\mathrm{CIC}$ users recruited in the longitudinal study $(n=56)$ meant that the logistic regression analysis was not statistically powered to allow us to draw strong conclusions about 
independent risk variables significantly associated with discontinuation. We had anticipated (from talking with clinicians) that a recommendation to undertake $\mathrm{CIC}$ following referral to the continence service would occur in around $50 \%$, whilst in fact it was only 34\% (69/204). Even more surprising was the number of participants in the longitudinal cohort who did not agree during the one-year follow-up to attempt $\mathrm{CIC}$, although recommended to do so $-13 / 69$ (19\%). This is an important group to follow-up in order to understand the transition from complete rejection of the idea of $\mathrm{CIC}$ to agreeing to try it, and is the focus of further research.

\section{Conclusion}

Although $\mathrm{CIC}$ may benefit many people with MS, continuation is dependent on the individual's perception of improvement in symptoms versus the burden of use. Using CIC can often be seen as 'giving in to the disease' and many require time to 'get their heads' around the idea. Symptom improvement, clinical and family support are important variables when the patient is learning the technique, however the most important factor that precipitates discontinuation is if the person links catheter use with the development of a UTI. The development of a UTI can have major health implications: it can negatively affect the person's MS, can take a long time to recover from fully, and can lead to feelings of guilt if the individual assumes they have caused the infection. Further research is required to determine if prophylactic use of antibiotics would prevent the development of UTIs during the learning stages of $\mathrm{CIC}$ keeping in mind the co-founding possibility of increasing urinary bacteria resistance. In addition, clinicians need to be able to identify when patients are ready and are available to offer the required support during the learning period. 


\section{References}

1. Tudor K, Sakakibara R, Panicker J. Neurogenic lower urinary tract dysfunction: evaluation and management J Neurol 2016: 263; 2555-2564 DOI 10.1007/s00415-016-8212-202.

2. Mahajan St, Patel PB, Marrie RA. Under treatment of overactive bladder symptoms in patients with multiple sclerosis: an ancillary analysis of the NARCOMS patient registry. J Urol. 2010: 183; 1432-1437

3. Fowler CJ, Panicker JN, Drake M, et al. 2008. A UK consensus on the management of the bladder in multiple sclerosis. J Neurol Neurosurg Psychiatry 2008:80; 470-7

4. Naish W. Intermittent self-catheterisation for managing urinary problems. Professional Nurse; 2003:18; 10, 585-587.

5. Newman $D$, Willson $M$. Review of intermittent catheterisation and current best practices. Urologic Nursing; 2011:31; 1, 12-48

6. McClurg D, Lowe-Strong A, Hagen S. 2008. Self-catheterisation for people with multiple sclerosis. Abstract accepted for presentation Enhancing Self Care - Helping People to Help Themselves. Social Dimensions of Health Institute. Abstract

7. Vahter $L$, et al. Clean intermittent self-catheterisation in persons with multiple sclerosis: the influence of cognitive dysfunction. Multiple Sclerosis. 2008: 00; 1-6

8. Creswell JW. 2014. Research Design. Sage. London

9. Jones and Bugge. Improving and understanding and rigour through triangulation: an exemplar based on patient participation in interaction. J Adv Nursing 2006:55(5); 612-21

10. Seth J, Haslam C, Panicker J. Ensuring patient adherence to clean intermittent self-catheterization. Patient Preference and Adherence 2014:8; 191-198

11. Peduzzi P, Concato J, Kemper E, Holford TR, Feinstein AR. A simulation study of the number of events per variable in logistic regression analysis. Journal of Clinical Epidemiology 1996:49; 13731379.

12. The EuroQol Group. EuroQol A new facility for the measurement of health-related quality of life. Health Policy 1990:16(3); 199-208.

13. Jackson S, Donovan J, Brooke S, Eckford S, Swithinbank L, Abrams, P. The Bristol Female Lower Urinary Tract Symptoms questionnaire: development and psychometric testing. BJU 1996:77; 805812

14. Donovan J, Peters T, Abrams P, Brooks S, de la Rosette J, Schafer W. 2000. Scoring the short form ICSmale SF questionnaire. J Uro 2000:164(6); 1948-1955

15. Brookes, S., Donovan, J., Wright, M., Jackson, S., Abrams, P. A scored form of the Bristol Lower Urinary Tract Symptoms questionnaire: data from a randomized controlled trial of surgery for women with stress incontinence. Am.J.Obstet.Gynecol. 2004:191(1); 73-82

16. Kelleher,C., Cardozo, L., Khullar,V., Salvatore, S. A new questionnaire to assess the quality of life of urinary incontinent women. BJOG. 1997:104; 1374-1379 
17. McClurg D, Hilton P, Dolan L, Hagen S, Dickinson L. Pelvic floor muscle training as an adjunct to prolapse surgery: a randomised feasibility study. Int Urogynecol J. 2014:25(7); 883-91. doi:

10.1007/s00192-013-2301-x.

18. Sacco, E., Sebastianelli, A., Batocchi, A. P., Pinto, F., Totaro, A., Volpe, A., Racioppi, M., Gulino, G., Bassi, P., Sexual dysfunctions in patients attected by multiple sclerosis: evaluation in a contemporary cohort from a referral center, Comunicazione, in EUROPEAN UROLOGY SUPPLEMENTS, (Vienna, 18-

22 March 2011), ELSEVIER SCIENCE BV, Amsterdam 2011: 160-160

[http://hdl.handle.net/10807/3573]

19. Guest G, Bunce A, Johnson L. How many interviews are enough? An experiment with data saturation and variablility. Field Methods 2006:18(1); 59-82

20. Ritchie J. \& Spencer L. 1994. Qualitative data analysis for applied policy research. In Analysing Qualitative Data (Bryman A. \& Burgess R.G. eds). Routledge, London, pp. 173-194.

21. O'Cathain A, Murphy E, Nicholl J. Three techniques for integrating data in mixed methods studies. BMJ 2010: 341c4587

22. Van Griensven H, Moore AP, Hall P. Mixed methods research - the best of both worlds? Manual Therapy 2014: doi 10.1016?j.math 2014-05005

23. Chartier-Kastler E, Denys P. Intermittent Cateterization with Hydrophylic Catheters as a Treatment of Chronic Neurogenic Urinary Retention. Neurourology and Urodynamics 2011: 30; 2131

24. Fumincelli L, Mazzo A, Martins J, Henriques F, Cardoso D, Rodrigues M. Quality of Life of Intermittent Urinary Catheterization Users and their Caregivers. Rev. Latino-AM. Enfermagem. 2017:25:e2906

25. Silva D. R., Mazzo A., Jorge B. M., Souza Júnior V. D., Fumincelli L., \& Almeida R. G. Intermittent Urinary Catheterization: The Impact of Training on a Low-Fidelity Simulator on the Self-Confidence of Patients and Caregivers. Rehabilitation Nursing, 2017:42(2); 97-103. doi: 10.1002/rnj.226

26. McClurg D, Walker K, Pickard R et al. Participant experiences of clean intermittest selfcatheterisation urinary tract infections and antibiotic use on the ANTIC trial - a qualitative study. Int J Nursing Studies 2018:18; 1-7 